\title{
Chaos and Quantum Chaos in Cosmological Models
}

\author{
R. GRAHAM \\ Fachbereich Physik, Universitaet GH Essen, Germany
}

\begin{abstract}
Spatially homogeneous cosmological models reduce to Hamiltonian systems in a low dimensional Minkowskian space moving on the total energy shell $H=0$. Close to the initial singularity some models (those of Bianchi type VIII and IX) can be reduced further, in a certain approximation, to a non-compact triangular billiard on a 2-dimensional space of constant negative curvature with a separately conserved positive kinetic energy. This type of billiard has long been known as a prototype chaotic dynamical system. These facts are reviewed here together with some recent results on the energy level statistics of the quantized billiard and with direct explicit semi-classical solutions of the Hamiltonian cosmological model to which the billiard is an approximation. In the case of Bianchi type IX models the latter solutions correspond to the special boundary conditions of a 'no-boundary state' as proposed by Hartle and Hawking and of a 'wormhole' state.
\end{abstract}

\section{INTRODUCTION}

The subject of chaos in cosmology is really an old one and predates the intense modern interest in chaotic dynamical systems. It started with the classical work of Belinsky et al 1969 [1] and of Misner 1969 [2] who found that the evolution of certain homogeneous cosmological models back into the past towards the 'Big Bang' shows an oscillatory behavior, some aspects of which in modern terminology one has to call chaotic. Useful references for these cosmological models are the books by Ryan 1972 [3] and Ryan and Shepley 1975 [4]. The chaotic aspects of their evolution have been examined in numerous papers starting with the review by Barrow 1982 [5]. Khalatnikov et al 1985 [6] give a detailed discussion. A dynamical systems approach was pioneered by Bogoyavlensky and Novikov 1973 [7] and elaborated by Ma and Wainwright 1989 [8]. Among the more recent works on this subject those of Pullin 1990 [9] and Rugh 1992 [10] may be mentioned. Most recently a Painlevé analysis was applied to this dynamical system by Cantopoulos et al 1993 [11] who found, surprisingly, that the system satisfies the Painlevé conditions which are necessary (but not sufficient) for a system to be integrable. 
Early on, again before the explosion of the interest in chaos took place, Misner 1972 [12] in a seminal paper and his student Chitre 1972 [13] in his thesis introduced coordinates in which the most interesting homogeneous cosmologies, those of Bianchi types VIII and IX, sufficiently close to the singularity reduce to a special two-dimensional billiard on a homogeneous space of constant negative curvature. The study of this kind of billiards is an even more ancient subject in mathematics, dating back to the work of Hadamard 1898 [14], Artin 1924 [15] and Hopf 1937 [16]. An extensive review is due to Balazs and Voros 1986 [17.

Given a dynamical system of Hamiltonian form the temptation to quantize seems to be irresistable. Hence the field of quantum chaos, which is concerned with the quantization of classically chaotic systems with the aim of understanding features of chaos and quantum mechanics, hence also the field of quantum cosmology, which starts with the quantization of spatially homogeneous cosmological models with the aim to achieve an understanding of quantum features of cosmology. For the Bianchi type IX cosmology this was first done by Misner 1972 in [12]. However, the present interest in quantum cosmology was mainly triggered by the proposal of a specific initial condition,

the 'no-boundary-state', by Hawking 1982 [18], and Hartle and Hawking 1983 [19]. Quantized versions of the homogeneous Bianchi type IX cosmological models were also investigated in this context, in semi-classical WKB-type approximations, e.g. in papers by Hawking and Luttrell 1984 [20], and Moss and Wright 1985 [21] who study consequences of the 'no-boundary'condition and by Del Campo and Vilenkin 1989 [22], and Graham and Szépfalusy 1990 [23] who instead pose the boundary condition of an outgoing wave. From the point of view of quantum chaos, the Bianchi IX model has also been studied further in papers by Furusawa 1986 [24] and Berger 1989 [25] who study the dynamics of wave-packets and by Graham et al. 1991 [26] and Csordás et al. 1991 [27] who study level statistics in Misner's billiard limit of the model. More recently further detailed studies of a class of billiards including the special one relevant to the Bianchi VIII and IX model have appeared in the quantum chaos literature (Bogomolny et al. 1992 [28, 29], Bolte et al. 1992 [30, Schmit 1991 [31] Eisele and Mayer 1993 [32]).

Finally, as the most recent development, exact solutions of quantized Bianchi IX models were found independently by Moncrief and Ryan 1991 [33], Graham 1991 [34], and Bene and Graham 1993 [35]. It is now understood that the solution found in the first two references describe virtual quantum wormholes (D.Eath 1993 [36], Bene and Graham 1993 [35]), while a further exact solution by Bene and Graham 1993 [35] gives the 'no-boundary-state' of the model in a simple analytical form.

The present paper has the purpose to review some of these results. In the next section we establish the necessary background on spatially homogeneous cosmological models. Then we proceed to consider Misner's approximate asymptotic reduction of the Bianchi VIII and Bianchi IX models to 2-dimensional billiards on a space of constant negative curvature, and discuss some results following from this reduction. Next, the Bianchi VIII and IX models are quantized, giving rise to their Schrödinger equation, called Wheeler DeWitt equation in the present context. We then discuss some of the properties of the billiard approximation of the quantized model. Finally, we turn to exact solutions of the Wheeler DeWitt equation and their physical interpretation.

\section{SPATIALLY HOMOGENEOUS COSMOLOGICAL MODELS}

We consider the class of space-times such that through every point there passes a space-like 3-manifold

which is left invariant by the actions of a three-dimensional Lie group (see e.g. Ryan and Shepley 1975 
[4]). Such space-times are called spatially homogeneous. The three-dimensional Lie groups have been classified by Bianchi into nine different types ('Bianchi types'). They can be distinguished by the Lie algebra of the group-generators, called 'Killing vectors' in general relativity. It is convenient to use an invariant (non-coordinate) basis and its dual of basis 1-forms $\omega^{i}$ on the invariant 3-manifold.

The space-time metric then takes the general form

$$
d s^{2}=-d t^{2}+g_{i j}(t) \omega^{i} \omega^{j}
$$

where $d t$ is the time-like one-form orthogonal to the invariant 3-manifold and $t$ is the standard cosmic time coordinate. The components $g_{i j}(t)$ of the 3-metric then depend only on $t$. The basis 1-forms satisfy

$$
d \omega^{i}=\frac{1}{2} C^{i}{ }_{j k} \omega^{j} \wedge \omega^{k}
$$

(we recall the usual definition $\omega^{j} \wedge \omega^{k}=\omega^{j} \otimes \omega^{k}-\omega^{k} \otimes \omega^{j}$ ) where the $C^{i}{ }_{j k}$ are the structure constants of the homogeneity group. Of special interest here will be the Bianchi type VIII with

$$
-C^{1}{ }_{23}=+C^{1}{ }_{32}=+C^{2}{ }_{31}=-C^{2}{ }_{13}=+C^{3}{ }_{12}=-C^{3}{ }_{21}=1
$$

and in particular the Bianchi type IX with

$$
C_{j k}^{i}=\epsilon_{i j k}
$$

In the latter case the homogeneity group is $S O(3)$, the invariant 3-manifold is compact and topologically a 3-sphere. This case therefore includes the closed Friedmann-Robertson-Walker (FRW) space-time and all homogeneous anisotropic modifications thereof. The invariant 3-manifolds of Bianchi-type VIII are non-compact and describe open space-times. (The open FRW space-times are not of this particular Bianchi-type, however, see Ryan and Shepley 1975 [4).

We shall restrict the further discussion to diagonal models, where $g_{i j}(t)$ can consistently be chosen diagonal,

$$
g_{i j}=\frac{1}{6 \pi} \operatorname{diag}\left(e^{\left(2 \alpha+2 \beta_{+}+2 \sqrt{3} \beta_{-}\right)}, e^{\left(2 \alpha+2 \beta+-2 \sqrt{3} \beta_{-}\right)}, e^{\left(2 \alpha-4 \beta_{+}\right)}\right)
$$

with three time-dependent parameters $\alpha, \beta_{+}, \beta_{-}$. The special space-time metric (1) can now be inserted in the action functional of general relativity, in particular in its first-order (Hamiltonian) form first given by Arnowitt, Deser, and Misner 1962 [37]. We shall neglect the matter contribution in the action, which, in the anisotropic case, can be shown to become unimportant close to the initial singularity. The result is a reduced action of the form

$$
S=\int d \lambda\left(p_{\nu} \dot{q}^{\nu}-N(\lambda) H(q, p)\right)
$$

with

$$
\begin{aligned}
N(\lambda) d \lambda & =\sqrt{\frac{3 \pi}{2}} e^{-3 \alpha(t)} d t \\
\dot{q}^{\nu} & =\frac{d q^{\nu}}{d \lambda} \\
H(q, p) & =\frac{1}{2}\left(-p_{\alpha}^{2}+p_{+}^{2}+p_{-}^{2}\right)+V\left(\alpha, \beta_{+}, \beta_{-}\right) .
\end{aligned}
$$

Here $N(\lambda)>0$ is a Lagrange parameter called the lapse function, whose variation enforces the condition

$$
H=0
$$


$q^{\nu}, p_{\nu}$ are used to denote the coordinates in the space of diagonal, homogeneous 3-metrics (minisuperspace) $\alpha, \beta_{+}, \beta_{-}$and their canonical momenta. The action (6) has the same form as for a particle in a 3-dimensional potential well, except for the Minkowskian nature of the metric in mini-superspace. The potential $V\left(\alpha, \beta_{+}, \beta_{-}\right)$is given by the scalar curvature ${ }^{(3)} R$ of the homogeneous 3 -metric

$$
V=-12 \pi^{2} \operatorname{det}\left(g_{i j}\right)^{(3)} R
$$

For the two Bianchi-types of special interest here it is given by (Ryan and Shepley 1975 [四)

$$
\begin{gathered}
V=\frac{1}{6} e^{4 \alpha}\left[2 e^{4 \beta_{+}}\left(\cosh \left(4 \sqrt{3} \beta_{-}\right)-1\right)+e^{-8 \beta_{+}}\right. \\
\left. \pm 4 e^{-2 \beta_{+}} \cosh \left(2 \sqrt{3} \beta_{-}\right)\right]
\end{gathered}
$$

where the upper and lower sign apply to Bianchi-type VIII and Bianchi-type IX, respectively.

The action (6) with eqs. (8)-(10) correctly generates the reduced equations of motion obtained from Einstein's equations with the ansatz (5). It therefore gives an exact description of the spatially homogeneous models.

We note that the potential (10) for Bianchi-type VIII is positive everywhere and decreases towards 0 for $\beta_{-}=0, \beta_{+} \rightarrow \infty$. It is therefore like a space-time dependent mass of a relativistic particle with $\alpha$ playing the role of the time-coordinate changing monotoneously with $\lambda$. For Bianchi-type IX, on the other hand, the potential is everywhere increasing for $\left|\beta_{+}\right| \rightarrow \infty$ or $\left|\beta_{-}\right| \rightarrow \infty$, and $V$ becomes negative in a neighborhood of the origin $\beta_{+}=\beta_{-}=0$. Therefore the action of $V$ cannot be compared to a mass-term in this case, and $\alpha$ need not change monotoneously with $\lambda$.

However, it can be shown (Bogoyavlensky and Novikov 1973 [7], Rugh 1992 [10]) that $\alpha$ cannot have a minimum $\frac{d \alpha}{d \lambda}=0, \frac{d^{2} \alpha}{d \lambda^{2}}>0$ and can have only a single maximum. To see this we use $H=0($ and $N=1)$ to express

$$
-\frac{d^{2} \alpha}{d \lambda^{2}}=-\frac{\partial H}{\partial \alpha}=-4 V=-2\left(\frac{d \alpha}{d \lambda}\right)^{2}+2 p_{+}^{2}+2 p_{-}^{2}
$$

which implies $d^{2} \alpha / d \lambda^{2}<0$ (except for $p_{+}=p_{-}=0$ )for $d \alpha / d \lambda=0$. Hence, sufficiently close to the singularity $\alpha \rightarrow-\infty$, where a maximum of $\alpha$ does not occur, $\alpha$ or any monotoneous function of $\alpha$ can in fact be used as a time-coordinate.

For $\alpha \rightarrow-\infty$ the potential (10) approaches zero for values of $\beta_{+}, \beta_{-}$inside a triangular region of the $\left(\beta_{+}, \beta_{-}\right)$-plane bounded by the three straight lines

$$
\begin{aligned}
& \alpha+\beta_{+} \pm \sqrt{3} \beta_{-}=0 \\
& \alpha-2 \beta_{+}=0
\end{aligned}
$$

The three sides of this triangle recede with decreasing 'time' $\alpha$ with velocity $1 / 2$. The velocity of the system point with respect to the time $\alpha$ in the region where $V$ is negligible is approximately 1 . The potential (10) rises steeply (exponentially) outside the lines (12) with the exception of narrow channels reaching to infinity. These channels are located symmetrically at all three corners of the triangle for Bianchi-type IX and in them the potential approaches zero from below for $\alpha \rightarrow-\infty$. For Bianchi-type VIII there is only one channel at the corner $\beta_{-}=0, \beta_{+}=-\alpha$ reaching to $\beta_{+}=+\infty$, and there the potential approaches zero from above. In an apparently reasonable approximation in the limit $\alpha \rightarrow-\infty$ the exponentially rising potential walls can be replaced by the infinitely steep walls of a billiard located 
at the straight lines (12). The picture of a billiard ball of unit velocity elastically bouncing from the walls of an equilateral triangle receding with velocity $1 / 2$ permits a thorough and lucid qualitative discussion of the resulting dynamics, which has been given in many papers and textbooks, starting with the classic works of Belinski et al 1969 [1] and Misner 1969 [2]. Detailed numerical simulations fully confirming the qualitative picture have recently been reported e.g. by Rugh 1992 [10]. We refer to the quoted literature for details. The channels at the corners, and the (for $\alpha \rightarrow-\infty$ increasingly) unlikely events, where the system point enters one of those channels, are not described by the billiard approximation. This fact makes it hard to assess the quality of the billiard approximation. In particular, chaos in the billiard approximation need not imply chaos in the unapproximated system, whose presence or absence is, in fact, still an open question (see Cantopoulos et al 1993 [11] for an indication that the system might be actually integrable; however, it should be noted that eq. (11) e.g. implies that the phase-space function $p_{\alpha} e^{-2 \alpha}$ varies monotonously with $\lambda$ which makes the system very special indeed, and calls for extreme caution before drawing too strong conclusions from the positive result of the Painlevé test for integrability).

In the next two sections we shall be concerned with the properties of the cosmological models in the billiard-approximation. However, in the final section we shall return to the description by the full Hamiltonian (8). The 'free'motion between two bounces is called a 'Kasner epoch' because the Kasner universe is described by the Hamiltonian (8) with $V \equiv 0$ (Ryan and Shepley 1975 [四). Repeated bounces between the same two walls constitute an 'era'. For $\alpha \rightarrow-\infty$, the motion then consists of an infinite sequence of 'eras' following each other in which the two walls defining each era are permutated in a chaotic manner (Barrow 1982 [5], Khalatnikov et al. 1985 [6]).

\section{REDUCTION TO A BILLIARD ON THE PSEUDO-SPHERE}

Discussion of chaos in the triangular billiard with moving walls are hampered by the fact that, with the use of $\alpha$ as an effective time-parameter, the system is explicitely time-dependent and the long-time limit $\alpha \rightarrow-\infty$, depending on its precise definition, is either trivial (free particle) or it does not exist (the particle-bounces never disappear). In fact an obvious but important point emphasized by Rugh 1992 [10] and Pullin 1990 [9] is the proper choice of the time-coordinate in discussions of chaos in general relativity: as Lyapunov exponents and Kolmogorov-Sinai entropy are not invariant under the free redefinitions of the time-coordinate permitted by general relativity some suitable coordinate condition must be posed in addition in order to preserve the usefulness of these mathematical concepts. Obviously one useful choice is a time variable which eliminates the explicit time-dependence at least asymptotically for sufficiently long time.

For the problem at hand such coordinates were provided by Misner 1972 [12], and first put to use in Chitre's thesis 1972 [13]. These coordinates $(t, \xi, \phi)$ are defined by

$$
\begin{aligned}
\beta_{+} & =e^{t} \sinh \xi \cos \phi \\
\beta_{-} & =e^{t} \sinh \xi \sin \phi \\
\alpha & =-e^{t} \cosh \xi \quad(\alpha<0) .
\end{aligned}
$$

Here $t$ is not to be confused with the synchronous time-coordinate in eq.(1). Rather, in the Minkowskian metric of the Hamiltonian (8) it is a time-like coordinate like $\alpha$. In fig. 1 we give a schematic plot illustrating the coordinate change. The triangular billiard walls (12) are shown in the $\left(\beta_{+}, \beta_{-}\right)$-plane for fixed $\alpha<0$. The corners of the triangle are on the circle $\beta_{+}^{2}+\beta_{-}^{2}=\alpha^{2}$. A fixed value of $\xi$ corresponds to 
Fig. 1: Billiard walls (12) and a circle $\xi=$ const in the $\left(\beta_{+}, \beta_{-}\right)$-plane for fixed $\alpha$.

the smaller circle $\beta_{+}^{2}+\beta_{-}^{2}=\alpha^{2} \tanh ^{2} \xi$. The corners of the triangle are therefore at $|\xi|=\infty$ in the new coordinates. The narrow potential channels at the corner of the Bianchi VIII and at all three corners of the Bianchi IX potentials can therefore not even be described in the new coordinates.

The billiard walls (12) in the new coordinates are at

$$
\tanh \xi=-\frac{1}{2} \sec \left(\phi+m \frac{2 \pi}{3}\right)
$$

with $m=0, \pm 1$. They are independent of the new variable $t$ which will be used as a new time coordinate instead of $\alpha$. The Hamiltonian (\$) expressed in the new coordinates and their canonical momenta takes the form

$$
\left.H=\frac{1}{2}\left(-p_{t}^{2}+p_{\xi}^{2}+\frac{p_{\phi}^{2}}{\sinh ^{2} \xi}\right) e^{-2 t}+V(\xi, \phi, t) .\right)
$$

Replacing the lapse function $N(\lambda)$ by

$$
N(\lambda) \rightarrow N(\lambda) e^{2 t}
$$

the Hamiltonian becomes

$$
H=\frac{1}{2}\left(-p_{t}^{2}+p_{\xi}^{2}+\frac{p_{\phi}^{2}}{\sinh ^{2} \xi}\right)+e^{2 t} V(\xi, \phi, t) .
$$

It still has to satisfy the condition $H=0$. For $t \rightarrow \infty$ the potential approaches 0 like $\exp \left(-\operatorname{const} . e^{t}+2 t\right)$, const. $>0$, inside the billiard walls (14), and it approaches infinity outside these walls. Therefore the Hamiltonian (17) ceases to be time-dependent in that limit and the canonical momentum $p_{t}$ is asymptotically conserved. The motion in this limit therefore becomes that of a free particle moving with arbitrary conserved energy $\frac{1}{2} p_{t}^{2}$ on a 2 -dimensional space with metric

$$
d l^{2}=d \xi^{2}+\sinh ^{2} \xi d \phi^{2}
$$

and bouncing elastically from the infinitely steep walls at the lines (14). The Riemannian space defined by eq. (18) has constant negative curvature, with Gaussian curvature $K=-1$ and Riemannian scalar curvature $R=-2$. Replacing $\xi$ by $r=\tanh \xi / 2$ it is represented by the pseudo-sphere $r \leq 1$ with

$$
d l^{2}=\frac{d r^{2}+r^{2} d \phi^{2}}{1-r^{2}}
$$


or by the Poincaré half-plane $\operatorname{Imz} \geq 0$ with

$$
\begin{aligned}
z & =\frac{\sqrt{3}}{2} i \frac{1-r e^{i\left(\phi+\frac{\pi}{6}\right)}}{1+r e^{i\left(\phi+\frac{\pi}{6}\right)}}-\frac{1}{2}=x+i y \\
d l^{2} & =\frac{d x^{2}+d y^{2}}{y^{2}} .
\end{aligned}
$$

It is obvious from eqs. (19), (20) that the metric in these coordinates is conformal to a Euclidean metric, i.e. all angles are as in a Euclidean space. The walls (14) are geodesics of the metric and define a triangular billiard with corners at infinity. Therefore the billiard is non-compact. However, its total metric area is finite. In the coordinates (20) the walls are at

$$
x=-1, x=0, y \geq 0 ;\left(x+\frac{1}{2}\right)^{2}+y^{2}=\frac{1}{4}, y \geq 0 .
$$

In these coordinates geodesics are semi-circles in the upper half of the $z$-plane with centers on the real axis. Due to the metric points on the real axis and points with $y=\infty$ are at infinity. For many purposes a geodesic which is repeatedly reflected on the walls is more conveniently represented on a somewhat enlarged domain, again bounded by geodesic walls, which are identified in opposite pairs corresponding to periodic boundary conditions. The smallest such domain preserving the geodesic is called the fundamental domain. It is shown in fig. 2 where the original billiard is displayed together with its extension to a fundamental domain. The letters $\mathrm{a}$ and $\mathrm{b}$ indicate which sides are to be identified. The conformal transformations identifying opposite sides together with the unit transformation generate elements of the 'Fuchsian group' of the billiard. Explicitely, these transformations are

$$
A: z^{\prime}=z+2 ; \quad B: z^{\prime}=\frac{z}{2 z+1}
$$

and their inverse. In order to return from the fundamental domain of the Fuchsian group to the original domain of the billiard, points connected by a reflection on the wall $x=0\left(z^{\prime}=-z^{*}\right)$ are identified.

Chitre 1972 [13] first used this construction to demonstrate ergodicity and mixing of the cosmological billiard invoking theorems by Hopf 1936 [16] and Hedlund 1939 [37] on geodesic flows on the quotient space of the pseudo-sphere and a Fuchsian group. For a similar non-compact billiard whose fundamental domain is the modular domain $|z|>1,-\frac{1}{2}<x<\frac{1}{2}, y>0$ with $A: z^{\prime}=z+1, B: z^{\prime}=-1 / z$ Artin 1924 [15] already gave a demonstration of ergodicity. He used the theory of continued fractions to show that the transformations of the Fuchsian group, applied to the real footpoints of a geodesic semi-circle, generate a sequence of footpoints which lie dense on the real axis (and hence generate semi-circles which lie dense in the modular domain) except for a set of footpoints of measure 0. The transformations (22) restricted to the real axis play an equally important role in the quantized billiard (see below).

Due to the fact that the walls are geodesics the Lyapunov exponent $\lambda$ and the Kolmogorov-Sinai entropy $h$ immediately follow from the equations for the geodesic deviations and are given by $\lambda=h=\left|p_{t}\right|$.

\section{QUANTIZATION}

The canonical quantization of the Hamiltonian (7) with the condition (8) yields the Wheeler DeWitt equation

$$
\left\{\frac{1}{2}\left(\left(\frac{\partial}{\partial \alpha}\right)^{2}-\left(\frac{\partial}{\partial \beta_{+}}\right)^{2}-\left(\frac{\partial}{\partial \beta_{-}}\right)^{2}\right)+V\left(\alpha, \beta_{+}, \beta_{-}\right)\right\} \psi\left(\alpha, \beta_{+}, \beta_{-}\right)=0
$$


Fig. 2: The cosmological triangular billiard (full lines) in the complex $z$-plane and its extension to a fundamental domain (dashed lines) with sides $a, a^{\prime}=A(a)$ and $b, b^{\prime}=B(b)$ pairwise identified.

Eq. (23) is particularly simple because the lapse function was chosen so as to make the metric in the variables $\left(\alpha, \beta_{+}, \beta_{-}\right)$flat. For a different choice of the lapse function the metric is no longer flat and its scalar curvature should appear in (23) together with the Laplace-Beltrami operator to render (23) invariant under such conformal transformations (Misner 1972 [12]). In the present section we wish to consider eq. (23) in the billiard approximation where it reduces to the quantized version of eq. (15) (whose lapse function is still the same as in (23)) with infinitely steep potential walls, i.e.

$$
e^{-2 t}\left[e^{-t} \frac{\partial}{\partial t}\left(e^{-t} \frac{\partial}{\partial t}\right)-\frac{1}{\sinh \xi} \frac{\partial}{\partial \xi}\left(\sinh \xi \frac{\partial}{\partial \xi}\right)-\frac{1}{\sinh ^{2} \xi} \frac{\partial^{2}}{\partial \phi^{2}}\right] \psi=0
$$

with Dirichlet boundary conditions on the lines (14). Separating the coordinate $t$ from $\xi, \phi$ by the ansatz

$$
\psi(t, \xi, \phi)=e^{-\left(i \omega+\frac{1}{2}\right) t} \psi(\xi, \phi)
$$

and introducing the coordinates (20) we obtain

$$
-y^{2}\left(\frac{\partial^{2}}{\partial x^{2}}+\frac{\partial^{2}}{\partial y^{2}}\right) \psi(x, y)=\left(\omega^{2}+\frac{1}{4}\right) \psi(x, y)
$$

with Dirichlet boundary conditions on the lines (21). We note that the eigenvalues $|\omega|$ give the allowed values of the observable $\left|p_{t}\right|$ which classically equals the Lyapunov exponent and the Kolmogorov Sinai entropy.

There is an immense mathematical literature on the eigenvalue problem (26), see Hejhal 1976 [39], Helgason 1981 [40, Venkov 1982 [41. One important result is that the eigenvalues $\omega$ of eq. (26) are given exactly by the zeros $s=\frac{1}{2}+i \omega$ of Selberg's zeta function, which is defined as an infinite product over the primitive classical periodic orbits, labelled by $p$, of geometric length $l_{p}$

$$
Z(s)=\prod_{p} \prod_{k}\left(1-\epsilon_{k}(p) \exp \left(-(s+k) l_{p}\right)\right)
$$


where $\epsilon_{k}(p)$ counts the number of reflections on the Dirichlet boundaries (21) and is given by $\epsilon_{k}(p)=1$ if that number is even, and $\epsilon_{k}(p)=-(-1)^{k}$ if that number is odd.

Another interesting observation, made by Bogomolny and Carioli 1992 [28], is the fact that the solution of eq. (26) can be reduced to the eigenvalue problem of a 1-dimensional quantum map. In general, the solutions $\psi(x, y)$ of eq. (26) have a representation in terms of functions $f(t)$ on the real line (Helgason 1981 [40])

$$
\psi(x, y)=\int_{-\infty}^{+\infty}\left(\frac{y}{t^{2}+y^{2}}\right)^{s} f(x+t) d t .
$$

which, like the $\psi$, transform according to an irreducible representation of the Fuchsian group $\Gamma: \psi_{s}(G(z))=$ $\chi(G) \psi_{s}(z) ; f(G(t))|D G(t)|^{1-s}=\chi(G) f(t) ; G \epsilon \Gamma$. Demanding, e.g., that $\psi$ transforms according to the unit representation of the Fuchsian group we find from (22)

$$
\begin{aligned}
& f(t)=f(t+2), f(t)=f(t-2) \\
& f(t)=f\left(\frac{t}{2 t+1}\right)|2 t+1|^{-1+2 i \omega} \\
& f(t)=f\left(\frac{t}{1-2 t}\right)|1-2 t|^{-1+2 i \omega} .
\end{aligned}
$$

The Dirichlet boundary conditions for $\psi$ are satisfied if $f(t)$ is restricted to odd functions

$$
f(t)=-f(t)
$$

Thus, $\omega$ and $f(t)$ are determined as eigenpair from the equation

$$
f(t)=f\left(\frac{t}{2 t+1}(\bmod 2)\right)|2 t+1|^{-1+2 i \omega}
$$

with the boundary condition (30). The $1-\mathrm{d} \operatorname{map} t^{\prime}=t /(2 t+1)(\bmod 2)$ appearing in eq. (31) is similar to the Gauss map. It appears as a one-dimensional map of the classical dynamics if the transformations $A, B$ eq. (22) are restricted to the real line. The 'eigenfunctions' $f(t)$ are rather singular objects (Helgason 1981 [40]) which makes it preferable to study the adjoint map induced by eq. (31) on the adjoints of $f(t)$ in a suitable scalar product. I refer to the papers by Bogomolny and Carioli 1992 [29], Mayer 1991 [42], and Eisele and Mayer 1993 [32] for further discussion.

The methods described so far are interesting in their own right, but they are not the most useful ones if one wishes to determine a large number of eigenvalues with rather high precision. This is done best by solving eq. (26) in a suitable representation directly on a computer (Csordás et al 1991 27], Schmit 1991 [31). The general solution of eq. (26) satisfying Dirichlet boundary conditions on $x=-1,0$ may be represented as

$$
\psi_{\omega}(x, y)=\sum_{n=1}^{\infty} A_{n} \sin (\pi n x) \sqrt{y} K_{i \omega}(\pi n y)
$$

Here

$$
K_{i \omega}(z)=\frac{1}{2} \int_{-\infty}^{\infty} e^{-z \cosh t+i \omega t} d t
$$

The Dirichlet condition on the remaining side $\left(x+\frac{1}{2}\right)^{2}+y^{2}=\frac{1}{4}$ yields

$$
0=\sum_{n=1}^{\infty} A_{n} \sin (\pi n x) \sqrt[4]{\frac{1}{4}-\left(x+\frac{1}{2}\right)^{2}} K_{i \omega}\left(\pi n \sqrt{\frac{1}{4}-\left(x+\frac{1}{2}\right)^{2}}\right) .
$$


The right-hand side can be expanded in the set $\{\sin (\pi n x)\}_{n=1}^{\infty}$ which is complete in $[-1,0]$ and we obtain

$$
D_{l}=\sum_{n=1}^{\infty} \mathrm{I}_{l n} A_{n}=0
$$

with

$$
\mathrm{I}_{l n}=4 F_{l} G_{n} \int_{-1}^{0} d x \sin (\pi l x) \sin (\pi n x) \sqrt[4]{\frac{1}{4}-\left(x+\frac{1}{2}\right)^{2}} K_{i \omega}\left(\pi n \sqrt{\frac{1}{4}-\left(x+\frac{1}{2}\right)^{2}}\right)
$$

where $F_{l}, G_{n}$ can be chosen arbitrarily, not zero, to simplify the eigenvalue condition

$$
\operatorname{det} \mathrm{I}_{l n}=0
$$

For the evaluation of the matrix elements (36) asymptotic expansions of the Hankel functions are used, which are given in Csordás et al 1991 [27]. The eigenfunctions of the Hamiltonian transform according to the irreducible representations of the triangle group $C_{3 v}$, which has two 1-dimensional irreducible representations corresponding to non-degenerate states and one two-dimensional representation. For the purposes of level statistics each symmetry class must be considered separately. Eigenfunctions transforming under the unit representation satisfy Neumann boundary conditions on the symmetry axes, those transforming under the second one-dimensional representation satisfy Dirichlet conditions there, instead. For these cases the original triangle (21) is replaced by the smaller triangle

$$
x=-\frac{1}{2}, x=0, y \geq 0 ; x^{2}+y^{2}=1,-\frac{1}{2}<x<0
$$

i.e. in the Fourier expansions (32)-(35) $n, l$ on the right hand side is replaced by $2 n, 2 l$ and

$$
\sqrt{\frac{1}{4}-\left(x+\frac{1}{2}\right)^{2}} \rightarrow \sqrt{1-x^{2}}
$$

In this way we restrict ourselves to a desymmetrized component of the original energy spectrum.

Remarkably, the only reason for the non-integrability of the Schrödinger eq. (26), (38) is the form of the boundary at $y=\sqrt{1-x^{2}}$ which breaks the translational invariance in $x$-direction. It is interesting therefore to unfold the billiard problem and to consider not only the boundary (39) but also the shifted boundary

$$
y=g\left(c_{p}, x\right)=1-\frac{1}{c_{p}}+\sqrt{\frac{1}{c_{p}^{2}}-x^{2}}
$$

depending on the parameter $c_{p}, 0 \leq c_{p} \leq 1$. The boundaries interpolate smoothly between the 'integrable' boundary $y=1$ for $c_{p}=0$, which approximates (38) in the interval $-\frac{1}{2} \leq x \leq 0$ reasonably well but preserves translational invariance, and the real boundary (38) for $c_{p}=1$. The integrable case $c_{p}=0$ has been investigated in Graham et al 1991 [26], the general case $c_{p}<1$ and $c_{p}=1$ in Csordás et al 1991 27] and, with emphasis on the transitions between the

different 'universality classes', further in Csordás et al 1993 43. We shall now proceed to discuss the level statistics obtained in Csordás et al 1991 [27]. 


\section{LEVEL STATISTICS}

A very useful check on the completeness of the energy levels $\lambda \equiv \omega^{2}+\frac{1}{4}$ obtained from eq. (37) up to a given energy $\lambda=\mu$ is provided by the smoothed level density as obtained from the semi-classical Weyl formula and its refinements. For the case at hand the Weyl formula was derived in Csordás et al 1991 [27. If $\bar{N}(\mu)$ is the smoothed number of eigenvalues $\lambda$ with $\lambda \leq \mu$, the Weyl formula reads

$$
4 \pi \bar{N}(\mu)=A \mu-\sqrt{\mu} \ln \mu-B \sqrt{\mu}+C+0\left(\mu^{-1 / 2}\right)
$$

with the area $A$ and the constants $B, C$ given as explicit functions of $c_{p}$. The term $-\sqrt{\mu} \ln \mu$ is due to the diverging circumference of the billiard. The numerically 'measured' function $N(\mu)$ deviates from $\bar{N}(\mu)$,

$$
N(\mu)=\bar{N}(\mu)+\frac{1}{2}-\delta N(\mu)
$$

In figs. 3 a,b, we plot $\delta N(\mu)$ for $c_{p}=1$ and $c_{p}=\frac{1}{2}$ for about 1400 levels, respectively. It can be seen that $\delta N(\mu)$ fluctuates around 0, indicating that no level has been missed (a systematic shift of the average of $\delta N(\mu)$ by 1 would be very noticeable in fig. 3). Another immediate observation from fig. 3 is that the fluctuations are significantly larger in the case $c_{p}=1$ than in the case $c_{p}=1 / 2$. Using the smoothed

Fig. 3: The deviation of the spectral staircase from the Weyl formula (a) $c_{p}=1$ and (b) $c_{p}=\frac{1}{2}$ (from Csordás et al [27]). 
level-staircase (41) the average level density can be scaled out by the transformation

$$
x_{i}=\bar{N}\left(\lambda_{i}\right)
$$

Then it is possible to determine the nearest neighbor level-spacing distribution $P(s) d s$ which gives the probability to find the next level $x_{i+1}$ in the interval $\left(x_{i}+s, x_{i}+s+d s\right)$ if there is a level at $x_{i}$. The integrated level spacing distributions $\mathrm{I}(s)=\int_{0}^{s} P(s) d s$ for $c_{p}=1, \frac{1}{2}$ are plotted in fig. 4 a,b, respectively, and compared with the Poissonian result (dashed curve)

$$
\mathrm{I}_{P}(s)=1-e^{-s}
$$

and the Wigner surmise for the Gaussian Orthogonal Ensemble (GOE) of

random matrix theory (dashed-dotted-curve)

$$
\mathrm{I}_{W}(s)=1-e^{-\pi s^{2} / 4} .
$$

For $c_{p}=1 / 2$ there is good agreement with the GOE result, indicating that the transition from the integrable to the chaotic case has occurred. However, the case $c_{p}=1$ fits the Poissonian distribution better than the GOE distribution, which was surprising at first (see Balazs and Voros 1986 [17] for the first observation of this phenomenon in a compact billiard), indicating some hidden non-generic behavior in this prototypical chaotic system. This non-genericity is now understood to be a consequence of hidden degeneracies occurring in some billiards on the pseudo-sphere which are due to certain special arithmetical properties of the underlying Fuchsian group (see below).

In fig. 5 a,b we also plot the averaged spectral rigidity

$$
\bar{\Delta}_{3}(L, x)=\frac{1}{p} \int_{x-p / 2}^{x+p / 2} \Delta_{3}\left(L, x^{\prime}\right) d x^{\prime}
$$

with

$$
\Delta_{3}(L, x)=\min _{A, B} \frac{1}{L} \int_{x-L / 2}^{x+L / 2}\left(N\left(x^{\prime}\right)-A x^{\prime}-B\right)^{2} d x^{\prime}
$$

again for $c_{p}=1,1 / 2$. For comparison the Poisson statistics $\bar{\Delta}_{3}=L / 15$ and the GOE statistics $\bar{\Delta}_{3} \simeq$ $\frac{1}{\pi^{2}} \ln L-0.00695 \ldots$ for large $L$ are also shown. For $c_{p}=1 / 2$ the GOE result fits the data well, while for $c_{p}=1$ the $\bar{\Delta}_{3}$-statistics for small $L$ is again closer to the Poissonian result. For larger $L$ the Poissonian result is not expected to be a good approximation, not even in the integrable case, because of the influence of periodic orbits (Berry 1985 [44]). For the integrable case $c_{p}=0$ see Graham et al 1991 [26]. The conclusion that the case $c_{p}=1$ is non-generic and similar to an integrable case is further confirmed by the $\Sigma_{2}$-statistics

$$
\Sigma_{2}=\left\langle\left[n_{i}(r)-\left\langle n_{i}(r)\right\rangle\right]^{2}\right\rangle
$$

where $n_{i}$ is the number of levels in an interval of size $r$. These data are shown in figs. 5 a, b respectively. An explanation for the strong deviation from GOE statistics in the chaotic case $c_{p}=1$ has been given by Bolte et al 1992 [30] and Bogomolny et al 1992 [28]. It is due to the existence of further symmetries of some of the Fuchsian groups (called 'arithmetic'), to which the infinite equilateral triangle also belongs. Classically these additional symmetries give rise to the appearance of a multiplicity $\langle g\rangle$ of periodic orbits of the same length $l$ with $\langle g\rangle$ for length $l<M$ growing exponentially with $M / 2$ for large $M$

$$
\langle g\rangle=\frac{\sum_{l<M} g(l)}{\sum_{l<M}}=\mathrm{const} \frac{e^{M / 2}}{M}
$$


When used, in Berry's 1985 [44]] semi-classical theory of level statistics the large value of $\langle g\rangle$ for $M>>$ 1 has consequences very similar to those of the infinite multiplicities of periodic orbits in integrable systems. Quantum mechanically for arithmetic Fuchsian groups there exists an infinite denumerable set of hermitian operators $T_{n}$ 'Hecke operators', commuting among themselves and with $H$, which can be diagonalized simultaneously with $H$ (Hejhal 1983 [39], Venkov 1990 [45]). Again one then expects Poissonian statistics, as observed. Even a slight shift of one of the walls $\left(c_{p}<1\right)$ is enough to destroy the length-degeneracy of the periodic orbits (or the conservation of the Hecke operators) and leads to a transition to GOE statistics for sufficiently high-lying levels. This transition has recently been studied in detail by Csordás et al 1993 [43], who found a scaling behavior in the variables $1-c_{p}$ and the energy quantum number $n$.

Fig. 4: The integrated nearest-neighbor level spacing distribution from approximately 1400 levels for (a) $c_{p}=1$ and (b) $c_{p}=\frac{1}{2}$. Solid, dashed, and dotted lines are the numerical result, the Poissonian, and the GOE predictions, respectively (from Csordás et al [27]). 
Fig. 5: The spectral rigidity $\Delta_{3}(L, x)$ at $x=700$ for (a) $c_{p}=1$ and (b) $c_{p}=\frac{1}{2}$. Solid, dashed, and dotted lines are the numerical result, the Poissonian, and the GOE predictions, respectively (from Csordás et al [27]).

\section{SEMI-CLASSICAL SOLUTIONS OF THE WHEELER DEWITT EQUATION}

The study of the separated eigenvalue euqation (26) presented in the two preceding sections has several drawbacks: (1) It can claim validity only in the asymptotic region $t \rightarrow+\infty$ where $t$ is the function of $\alpha, \beta_{+}^{2}+\beta_{-}^{2}$ defined in eq. (13). (2) It completely neglects the influence of the potential channels at the corners of the triangle in configuration space, which become extremely narrow for $t \rightarrow+\infty$, but, in principle remain always open. (3) Even if eq. (26) gave a complete description it would leave open the question which linear superposition of the eigenstates of (26) determines the actual wave-function of the Universe.

For all these reasons it seems desirable to avoid the transformation (13) and instead to go back to the original Wheeler DeWitt equation (23), even at the cost of treating a more difficult problem.

As we are still interested in the asymptotic region where the influence of matter is negligible we may anticipate that the solution of (23) will have the form of a bound state

$$
\psi\left(\alpha, \beta_{+}, \beta_{-}\right) \sim \exp \left(-\phi\left(\alpha, \beta_{+}, \beta_{-}\right) / \hbar\right)
$$

where we restore Planck's constant here and in (23) in order to have a formal control over the semiclassical limit $\hbar \rightarrow 0$. Surprisingly, exact analytical solutions of the Wheeler DeWitt equation of the form (50) can 
Fig. 6: $\Sigma_{2}(r)$ statistics for (a) $c_{p}=1$ and (b) $c_{p}=\frac{1}{2}$. Solid, dashed, and dotted lines are the numerical result, the Poissonian, and the GOE predictions, respectively (from Csordás et al [27]).

be given (Graham 1991 [34], Moncrief and Ryan 1991 [33] Bene and Graham 1993 [35]). The potential $\phi$ in eq. (50) may be interpreted as a Euclidean action which is required for the system in its bound state to tunnel from some initial point $\alpha_{0}, \beta_{+0}, \beta_{-0}$ to a given end-point $\alpha, \beta_{+}, \beta_{-}$. (In this context it may be helpful to recall a harmonic oscillator with coordinate $x$ in its ground state where in appropriate units $\phi=x^{2}$ is the Euclidean action for tunnelling from $x=0$ to the classically forbidden amplitude $x \neq 0$ ).

Inserting the ansatz (50) in eq. (23) (and taking $\hbar \rightarrow 0$ or adding suitable terms of order $\hbar$ to the potential $V$ which is always possible by an appropriate choice of factor ordering when defining the 
quantized model, See Moncrief and Ryan 1990 [32]) we find the Euclidean Hamilton Jacobi equation [?

$$
-\frac{1}{2}\left(\frac{\partial \phi}{\partial \alpha}\right)^{2}+\frac{1}{2}\left(\frac{\partial \phi}{\partial \beta_{+}}\right)^{2}+\frac{1}{2}\left(\frac{\partial \phi}{\partial \beta_{-}}\right)^{2}-V\left(\alpha, \beta_{+}, \beta_{-}\right)=0
$$

which now contains the inverted potential $-V$. The following exact analytical solutions of this Euclidean Hamilton Jacobi equation are known, Belinsky et al 1978 [46], Gibbons and Pope 1979 [47, Atiyah and Hitchin 1985 48, in the case of Bianchi type IX

$$
\begin{aligned}
\phi_{1} & =\frac{1}{6}\left(e^{2 \beta_{+}+2 \sqrt{3} \beta_{-}}+e^{2 \beta_{+}-\sqrt{3} \beta_{-}}+e^{-4 \beta_{+}}\right) \\
\phi_{2} & =\phi_{1}-\frac{1}{3} e^{2 \alpha}\left(e^{2 \beta_{+}}+e^{-\beta_{+}+\sqrt{3} \beta_{-}}+e^{-\beta_{+}-\sqrt{3} \beta_{-}}\right) \\
& =V\left(\frac{\alpha}{2}, \frac{\beta_{+}}{2}, \frac{\beta_{-}}{2}\right) \\
\phi_{3} & =\phi_{1}-\frac{1}{3} e^{2 \alpha}\left(e^{2 \beta_{+}}-e^{-\beta_{+}+\sqrt{3} \beta_{-}}-e^{-\beta_{+}-\sqrt{3} \beta_{-}}\right) .
\end{aligned}
$$

From $\phi_{3}$ two other solutions may be obtained by $120^{\circ}$ degree rotations in the $\left(\beta_{+}, \beta_{-}\right)$-plane. It is clear that $-\phi$ is also a solution if $\phi$ is a solution. In the following we shall only consider the solutions $\phi_{1}, \phi_{2}$ which have the full triangular symmetry of the problem. In figs. 6 and 7 we give plots of the wavefunctions (50) obtained with $\phi_{1}, \phi_{2}$ for different fixed values of $\alpha$. The maximum $\psi$ is always normalized to unity. It is seen that the channels indeed modify the wave-function. However, because the $\phi_{i}$ are real there is only a tunnelling penetration into the channels without any real propagation inside them.

For the case of Bianchi type VIII very similar analytical solutions exist. They are given by (Bene and Graham 1993 [35)

$$
\begin{aligned}
\phi_{1} & =\frac{1}{6} e^{2 \alpha}\left(e^{2 \beta_{+}+2 \sqrt{3} \beta_{-}}+e^{2 \beta_{+}-2 \sqrt{3} \beta_{-}}-e^{-4 \beta_{+}}\right) \\
\phi_{2} & =\frac{1}{6} e^{2 \alpha}\left(4 e^{2 \beta_{+}} \sinh ^{2} \sqrt{3} \beta_{-}-e^{-4 \beta_{+}} \pm 4 i e^{-\beta_{+}} \cosh \sqrt{3} \beta_{-}\right) \\
\phi_{3} & =\frac{1}{6} e^{2 \alpha}\left(4 e^{2 \beta_{+}} \cosh ^{2} \sqrt{3} \beta_{-}-e^{-4 \beta_{+}} \pm 4 i e^{-\beta_{+}} \sinh \sqrt{3} \beta_{-}\right)
\end{aligned}
$$

The fact that $\phi_{2}$ and $\phi_{3}$ turn out to be complex is due to the non-binding nature of the Bianchi VIII potential which now makes a real propagation of the wave-function (without tunnelling) inside the channel along the positive $\beta_{+}$-axis possible and gives rise to a real physical action (i.e. an imaginary Euclidean action). For $\alpha \rightarrow-\infty$ and values of $\beta_{+}, \beta_{-}$inside the triangle (11) the imaginary part of $\phi$ is negligibly small. Yet solutions of the form (50) with $\phi= \pm \phi_{j}, \phi_{j}$ of eq. (53), turn all out to be non-normalizable for fixed $\alpha$, i.e. none of these solutions is physically acceptable for Bianchi type VIII.

We now turn to the problem of physically interpreting the special solutions (52). First of all, this involves the question of the interpretation of the wave-function of the Universe, which would need a discussion we don't wish to enter here (see e.g. the discussion in Bene and Graham 1993 [35]). We

${ }^{*}$ A Euclidean Hamilton Jacobi equation (51) with a potential $V_{0}\left(\alpha, \beta_{+}, \beta_{-}\right)$via eq. (50) corresponds to a Wheeler DeWitt equation (23) with a potential

$$
V=V_{0}\left(\alpha, \beta_{+}, \beta_{-}\right)+\frac{\hbar}{2}\left(\frac{\partial^{2} \phi}{\partial \alpha^{2}}-\frac{\partial^{2} \phi}{\partial \beta_{-}^{2}}-\frac{\partial^{2} \phi}{\partial \beta_{-}^{2}}\right)
$$

Such a potential appears naturally in supersymmetric formulations (Graham 1991 [33, Bene and Graham 1993 [36]). However, in any case $V$ is reduced to $V_{0}$ in the semiclassical limit $\hbar \rightarrow 0$. 
Fig. 7: Wormhole state: The wave function $|\psi|^{2}$ for $\phi=\phi_{1}$ as a function of $\beta_{+}, \beta_{-}$for four different values of $\alpha$. The maximum of $|\phi|^{2}$ is always normalized to 1 . The changing scale of $\beta_{+}, \beta_{-}$should be noted (after Graham 1991 [34]). 
Fig. 8: No-boundary (Hartle- Hawking) state: The same as Fig. 7 but for $\phi=\phi_{2}$. 
shall simply interpret $|\psi|^{2}$ for fixed logarithm of the scale parameter $\alpha$, as the probability distribution of the anisotropy parameters $\beta_{+}, \beta_{-}$for that scale parameter. However, we still have to understand which wave-function applies to which physical situation. This question can be answered by recalling the meaning of $\phi$ as a Euclidean action. The (imaginary-time) solutions of the classical equations of motion corresponding to these actions can be interpreted (for $\hbar \rightarrow 0$ ) as the most probable paths followed in the tunnelling propcess. These paths, in the present case, correspond to a sequence of 3 -spaces followed in the tunnelling process from a given intial 3-space up to the final 3 -space whose parameters $\alpha, \beta_{+}, \beta_{-}$ appear as the arguments of $\phi$. The initial point of the tunnelling path is chosen as a most probable state $\phi=\min$ for $\alpha$ fixed where the system likes to sit when it does not fluctuate away on one of its tunnelling excursions to the neighborhood (again, recall the harmonic oscillator in its ground state for a simple example of this physical picture). Let us now consider the most likely tunnelling paths. They satisfy

$$
\begin{aligned}
& p_{\alpha}=-\frac{d \alpha}{d \lambda}=\frac{\partial \phi}{\partial \alpha}=2 \phi \\
& p_{+}=\frac{d \beta_{+}}{d \lambda}=\frac{\partial \phi}{\partial \beta_{+}} \\
& p_{-}=\frac{d \beta_{-}}{d \lambda}=\frac{\partial \phi}{\partial \beta_{-}}
\end{aligned}
$$

It follows that

$$
\begin{aligned}
& \frac{d \beta_{+}}{d \alpha}=-\frac{1}{2} \frac{\partial}{\partial \beta_{+}} U\left(\beta_{+}, \beta_{-}\right) \\
& \frac{d \beta_{-}}{d \alpha}=-\frac{1}{2} \frac{\partial}{\partial \beta_{-}} U\left(\beta_{+}, \beta_{-}\right)
\end{aligned}
$$

with the new $\alpha$-independent potential

$$
U\left(\beta_{+}, \beta_{-}\right)=\ln \left|e^{-2 \alpha} \phi\left(\alpha, \beta_{+} \cdot \beta_{-}\right)\right|
$$

According to eq. (55) the evolution of $\beta_{+}, \beta_{-}$with increasing $\alpha$ is on lines of steepest descent of the potential $U$.

Let us now consider the concrete Bianchi type IX solutions $\phi_{1}$ and $\phi_{2}$ of eq. (52). For $\phi=\phi_{1}$ eqs. (55) can be solved explicitely (Belinsky et al 1978 [46]) yielding the Riemannian space-time metric

$$
d s^{2}=\frac{d \rho^{2}}{\sqrt{F(\rho)}}+\frac{1}{4} \rho^{6} \sqrt{F(\rho)}\left(\frac{\omega^{1} \omega^{1}}{\rho^{4}-a_{1}^{4}}+\frac{\omega^{2} \omega^{2}}{\rho^{4}-a_{2}^{4}}+\frac{\omega^{3} \omega^{3}}{\rho^{4}-a_{3}^{4}}\right)
$$

with

$$
F(\rho)=\left(1-\frac{a_{1}^{4}}{\rho^{4}}\right)\left(1-\frac{a_{2}^{4}}{\rho^{4}}\right)\left(1-\frac{a_{3}^{4}}{\rho^{4}}\right)
$$

where $a_{1}, a_{2}, a_{3}$ are constants of integration which must be chosen in such a way that the spatial 3-metric of eq. (57) becomes equal to the Bianchi type IX 3 -metric with given $\alpha, \beta_{+}, \beta_{-}$for a suitable choice of $\rho$. It follows from (57) that the Euclidean coordinate time $(i t)$ is $d(i t)=(F(\rho))^{-1 / 4} d \rho$. The solution (57) must be restricted to $\rho^{2}>\max \left(a_{1}^{2}, a_{2}^{2}, a_{3}^{2}\right)$ otherwise the 3-geometry is separated from the initial 3-geometry by a singularity.

The tunnelling path starts at the minimum of $\phi_{1}$ for fixed $\alpha$, which is at $\beta_{+}=0=\beta_{-}$, corresponding to $\rho=\infty$ in eq. (57). The given Bianchi-IX metric corresponds to the inner boundary at small $\rho^{2}$ (but $\left.\rho^{2}>\max \left(a_{1}^{2}, a_{2}^{2}, a_{3}^{2}\right)\right)$. For $\rho \rightarrow \infty$ the 4-metric (57) approaches $d s^{2}=d \rho^{2}+\frac{1}{4} \rho^{2}\left(\omega^{1} \omega^{1}+\omega^{2} \omega^{2}+\omega^{3} \omega^{3}\right)$, i.e. it 
becomes Euclidean and flat (Belinsky et al 1978 [46]), indicating that the wave-function under discussion here describes the spontaneous quantum fluctuation of a flat 3-metric to one with an anisoptropic inner boundary or throat, i.e. a quantum wormhole (D'Eath 1993 [36], Bene and Graham 1993 [35]).

Turning now to $\phi=\phi_{2}$ we note that a transparent closed-form solution like (57) is not available in this case. However, we may use eqs. (55) and the first of eqs. (54) to obtain the complete physical picture. Let us note that for $\phi=\phi_{2}$ the equi-potential lines of $\phi_{2}$ and of $U\left(\beta_{+}, \beta_{-}\right)$coincide with those of $V\left(\alpha, \beta_{+}, \beta_{-}\right)$for fixed $\alpha$. The minimum of $\phi_{2}$ for fixed $\alpha$ is again at $\beta_{+}=0=\beta_{-}$defining the most likely starting point of the tunnelling path. This point corresponds to a local isotropic maximum of $U$, i.e. if $\beta_{+}, \beta_{-}$beginn to tunnel to small non-zero values, $\alpha$ increases from its inital value $-\infty$ meaning that the Universe expands from nothing in some random anisotropic way. From the first of eqs. (54) it follows that this expansion occurs with increasing $\lambda$, since $\phi_{2}(\alpha, 0,0)<0$. However, $U\left(\beta_{+}, \beta_{-}\right)$has a degenerate minimum on the line where $V\left(\alpha, \beta_{+}, \beta_{-}\right)$(and hence also $\left.\phi_{2}\right)$ change sign. In fact this line is formed by a triangle of three hyperbolas which are asymptotic to the positive $\beta_{+}$axis and the two axes obtained by $\pm 120^{\circ}$ rotations. Outside this triangle of hyperbolas the equipotential lines of $V, \phi_{2}$ and $U$ are no longer closed due to the existence of the channels on the three axes. Linearizing eqs. (55) in the neighborhood of the line of minimal $U$ one finds that with increasing $\lambda$ the line is in fact crossed, but while crossing $\alpha$ reaches a maximum and decreases again as $\beta_{+}, \beta_{-}$start climbing the potential $U\left(\beta_{+}, \beta_{-}\right)$outside the triangle of hyperbolas to their given final value.

Thus for given values of $\left(\beta_{+}, \beta_{-}\right)$outside (or inside) the said triangle of hyperbolas the most probable path of tunnelling starts with vanishing 3-volume $(\alpha=-\infty)$ and goes (or, respectively, does not go) through a maximum of the 3 -volume until it reaches the given values $\alpha, \beta_{+}, \beta_{-}$. The given 3 -metric in this case forms the outer (and in fact the only) boundary of the compact Riemannian space-time region traced out by the 3-geometries along the most probable tunnelling path, and $\phi_{2}$ is the Euclidean action of this compact space-time region. Hartle and Hawking 1983 [19] made the general proposal that in the semi-classical limit the wave-function of the universe is given as the exponential of the Euclidean action constructed in this way. Thus

$$
\psi=\text { const } \exp \left(-\phi_{2}\left(\alpha, \beta_{+}, \beta_{-}\right) / \hbar\right)
$$

is identified as the no-boundary state of the Bianchi-type IX quantum space-time (Bene and Graham 1993 [35]). The state has also been discussed by Hawking and Luttrell 1984 [20] and by Moss and Wright 1985 [21], however, this work was qualitative or numerical and the analytical expression (59) was not given there.

A similar discussion for $\phi_{3}$ of eq. (52) shows that the wave-function (50) for this case arises by quantum tunnelling from a disk shaped 3-geometry $\left(\beta_{+}=\infty, \beta_{-}=0\right)$ with infinite volume $(\alpha \rightarrow \infty)$ down to a given 3-geometry $\alpha, \beta_{+}, \beta_{-}$with $\alpha$ decreasing monotonously along the most probable tunnelling path which closely follows the $\beta_{+}$-axis before it finally turns to the given value $\beta_{-}$. This is again a solution of wormhole type, but this time of a virtual wormhole in a highly anisotropic 3-geometry.

Finally, let us compare the solutions obtained in the present section with the solutions obtained in the two preceding sections. First, we note that the solutions given there have only restricted validity, as they were based on an approximation which is only valid in the limit $\alpha \rightarrow-\infty$. On the other hand, in that limit, the Wheeler DeWitt equation, has been solved there, in principle, as a general linear superposition of the solutions of the eigenvalue problem of the two-dimensional billiard. In order to select the special cosmologically relevant solution an additional 'initial' or boundary condition would still be needed. The simplest choice is to select the ground state of the billiard (Graham and Szépfalusy 1990 [23]), but other 
choices may, of course, be possible. By contrast, in the present section we have obtained some particular solutions only, which are, however, valid for arbitrary $\alpha$. As discussed above, these solutions correspond to the special boundary conditions of the 'no-boundary state' and the wormhole state. Their cosmological significance is therefore clear. If specialized to the regime $\alpha \rightarrow-\infty$ these particular solutions may be represented as linear superpositions of the eigenmodes of the two-dimensional billiard, i.e. they can be used to determine the probability amplitudes of the eigenmodes of the billiard in the wave-function of the Universe. As can be seen from figs. 6, 7 for $\alpha \rightarrow-\infty$ not only the ground state of the billiard, but also arbitrarily high lying states will be excited, due to the increasingly sharp drop of the wave-function from a nearly constant value inside the billiard to 0 at the boundary. In fact it appears that these solutions asymptotically approach the constant solution of the billiard for Neumann boundary conditions which contains arbitrarily high lying states satisfying Dirichlet boundary conditions. The kinetic energy of the billiard is the positive energy residing in the anisotropy degrees of freedon $\beta_{+}, \beta_{-}$or $x, y$. This positive energy is exactly balanced, because of $H=0$, by the negative energy $-\frac{1}{2} p_{t}^{2}$ of gravitational attraction. The particular solutions discussed in the present section, in the limit $\alpha \rightarrow-\infty$, contain components for which both energies are unbounded.

\section{ACKNOWLEDGEMENT}

I am very greatful to Peter Szépfalusy for first stimulating my interest in the quantum mechanics of the mixmaster cosmology. The results on the level statistics were obtained in joint work with P. Szépfalusy, A. Csordás, G. Vattay and R. Hübner. The work reported in the last section was done, in part, in collaboration with J. Bene. All those mentioned I would like to thank for a most enjoyable and fruitful collaboration. Finally I would like to thank Peter D'Eath and Gary Gibbons for enlightening discussions concerning the topics of the last section.

This work was supported by the Deutsche Forschungsgemeinschaft through the Sonderforschungsbereich 237 'Unordnung und große Fluktuationen' and by the 'Deutsch-Ungarisches Kooperationsabkommen' through grant X231.3.

\section{References}

[1] V. A. Belinsky, I. M. Khalatnikov, Zh. Eksp. Teor, Fiz. 56, 1700 (1969), Sov. Phys. JETP 29, 911 (1969); Zh. Eksp. Teor, Fiz. 57, 2163 (1969), Sov. Phys. JETP 30, 1174 (1970); Zh. Eksp. Teor, Fiz. 59, 314 (1970), Sov. Phys. JETP 32, 169 (1970); V. A. Belinsky, I. M. Khalatnikov, and E. M. Lifshitz, Usp. Fiz. Nauk 102, 463 (1970), Advances in Physics 19, 525 (1970); V. A. Belinsky, E. M. Lifshitz, and I. M. Khalatnikov, Zh. Eksp. Teor. Fiz. 60, 1969 (1971), Sov. Phys. JETP 33, 1061 (1971).

[2] C. W. Misner, Phys. Rev. Lett. 22, 1071 (1969).

[3] M. P. Ryan, Jr., 'Hamiltonian Cosmology', (Springer, Berlin 1972).

[4] M. P. Ryan, Jr., and L. C. Shepley, 'Homogeneous Relativistic Cosmologies', (Princeton University Press, 1975).

[5] J. D. Barrow, Phys. Rep. 85, 1 (1982). 
[6] I. M. Khalatnikov, E. M. Lifshitz, K. M. Khanin, I. Schur, and Ya. G. Sinai, J. Stat. Phys. 38, 97 (1985).

[7] O. I. Bogoyavlensky and S. P. Novikov, Zh. Eksp. Teor. Fiz. 64, 1475 (1973), Sov. Phys. JETP 37, 747 (1973).

[8] P. K. H. Ma and J. Wainwright, 'A Dynamical Systems Approach to the Oscillatory Singularity in Bianchi Cosmologies', preprint 1990.

[9] J. Pullin, 'Time and Chaos in General Relativity', Syracuse University preprint 90-0734 (1990).

[10] S. E. Rugh, 'Chaos in the Fundamental Forces-Characterization and Importance?', Licentiate Thesis, The Niels Bohr Institute 1992.

[11] G. Cantopoulos, B. Grammaticos and A. Ramani, 'Painleveé Analysis for the Mixmaster Model', preprint 1993.

[12] C. W. Misner, in Magic without Magic, Ed. Klauder, J., Freeman, San Francisco (1972).

[13] D. M. Chitre, University of Maryland Technical Report No. 72-125, (1972).

[14] J. Hadamard, J. de Math. Pures et Appl. 4, 27 (1898).

[15] E. Artin, Hamburger Math. Abh. 3, 170 (1924).

[16] E. Hopf, 'Ergodentheorie', (Springer, Berlin 1937).

[17] N. L. Balazs and A. Voros, Phys. Rep. 143, 109 (1986).

[18] S. W. Hawking, in 'Astophysical Cosmology', ed. H. A. Bruck et al. , (Pontoficia Academiae Scientiarum, Vatican 1982).

[19] J. B. Hartle and S. W. Hawking, Phys. Rev. D28, 2960 (1983).

[20] S. W. Hawking, J. C. Luttrell, Phys. Lett. 143B, 83 (1984).

[21] W. A. Wright, I. G. Moss, Phys. Lett. 154B, 115 (1985).

[22] S. Del Campo, A. Vilenkin, Phys. Lett. 224B, 45. (1989)

[23] R. Graham, P. Szépfalusy, Phys. Rev. D42, 2483 (1990).

[24] T. Furusawa, Progr. Theor. Phys. 75, 59 (1986); Progr. Theor. Phys. 76, 67 (1986).

[25] B. K. Berger, Phys. Rev. D39, 2426 (1989).

[26] R. Graham, R. Hübner, P. Szépfalusy, G. Vattay, Phys. Rev. A44, 7002 (1991).

[27] A. Csordás, R. Graham, P. Szépfalusy, Phys. Rev. A44, 1491 (1991).

[28] E. B. Bogomolny, B. Georgot, M.-J, Giannoni, and C. Schmit, Phys. Rev. Lett. 69, 1477 (1992).

[29] E. B. Bogomolny and M. Carioli, 'Quantum Maps from Transfer Operators', (Physica D 1992). 
[30] J. Bolte, G. Steil, and F. Steiner, Phys. Rev. Lett. 69, 2188 (1992).

[31] C. Schmit, 'Triangular Billiards on the Hyperbolic Plane: Spectral Properties', IPNO/TH 91-68, 1991.

[32] M. Eisele and D. Mayer, 'Dynamical zeta functions for Artin's billiard and the Venkov-Zograf factorization formula', preprint 1993.

[33] V. Moncrief, M. P. Ryan, Jr., Phys. Rev. D44, 2375 (1991).

[34] R. Graham, Phys. Rev. Lett. 67, 1381 (1991); Phys. Lett. 277B, 393 (1992)

[35] J. Bene and R. Graham, Phys. Rev. D (1993).

[36] P. D. D'Eath, Phys. Rev. D48, 713 (1993).

[37] R. Arnowitt, S. Deser, and C. W. Misner, in 'Gravitation: An Introduction to Current Research', ed. L. Witten, (Wiley, New York 1962).

[38] G. A. Hedlund, Bull. Am. Math. Soc. 45, 241 (1939).

[39] D. A. Hejhal, 'The Selberg Trace Formula for $P S L(2, R)^{\prime}$, Springer Lecture Notes in Math. 548, (1976); 1001, (1983).

[40] S. Helgason, 'Topics in Harmonic Analysis on Homogeneous Spaces', (Birkhäuser, Basel 1981), Chap. 4.

[41] A. B. Venkov, Usp. Math. Nauk. 34, 69 (1979), Russ. Math. Surveys 34, 79 (1979).

[42] D. Mayer, Bull. Amer. Math. Soc. 25, 55 (1991); in 'Ergodic Theory, Symbolic Dynamics and Hyperbolic Spaces', eds. T. Bedford et al. (Oxford University Press, Oxford 1991); in 'From Phase Transition to Chaos', eds. G. Györgyi et al. (World Scientific, Singapore 1992).

[43] A. Csordás, R. Graham, P. Szépfalusy, G. Vattay, Phys. Rev. A, (1993).

[44] M. V. Berry, Proc. Roy. Soc. London A Ser. 400, 229 (1985).

[45] A. B. Venkov, 'Spectral Theory of Automorphic Functions and its Applications', (Kluwer, Dordrecht 1990).

[46] V. A. Belinsky, G. W. Gibbons, D. N. Page, and C. N. Pope, Phys. Lett. 76B, 433 (1978).

[47] G. W. Gibbons and C, N. Pope, Comm. Math. Phys. 66, 267 (1979).

[48] M. F. Atiyah and N. J. Hitchin, Phys. Lett. 107A, 21 (1985). 
This figure "fig1-1.png" is available in "png" format from: http://arxiv.org/ps/gr-qc/9403030v2 
This figure "fig2-1.png" is available in "png" format from: http://arxiv.org/ps/gr-qc/9403030v2 
This figure "fig1-2.png" is available in "png" format from: http://arxiv.org/ps/gr-qc/9403030v2 
This figure "fig2-2.png" is available in "png" format from: http://arxiv.org/ps/gr-qc/9403030v2 
This figure "fig1-3.png" is available in "png" format from: http://arxiv.org/ps/gr-qc/9403030v2 
This figure "fig2-3.png" is available in "png" format from: http://arxiv.org/ps/gr-qc/9403030v2 
This figure "fig2-4.png" is available in "png" format from: http://arxiv.org/ps/gr-qc/9403030v2 